\title{
Screening for Cytomegalovirus Infection During Pregnancy in a Teaching Hospital, Western province, Sri Lanka
}

\author{
Abeynayake J I a , Perera MAK ${ }^{b}$, Gunasekara C K ${ }^{c}$, Samaraweera B ${ }^{\mathrm{d}}$, Wickramasinghe $\mathrm{N}^{\mathrm{e}}$
}

\begin{abstract}
Primary Cytomegalovirus (CMV) infection during pregnancy is a serious threat to the fetus. Although vertical transmission is likely to occur as a result of maternal infection, the rate of permanent sequelae is higher among infants born to mothers with primary CMV infection. Diagnosis of CMV infection among healthy adults generally relies on serological testing as most of them are asymptomatic. CMV infection in pregnancy is barely studied in Sri Lanka. This study aims in screening for CMV infection of selected pregnant population in a major maternity hospital and to describe associated factors with seropositivity. This study included 385 pregnant women in second trimester presented during a period of 6 months. Mothers with immunocompromised states were not recruited. Questionnaire was used to gather sociodemographic and clinical factors. All serum samples were tested for CMV$\mathrm{IgG}$ and IgG-avidity assay for seropositives. CMV-IgM test was carried out on samples with negative CMV-IgG and low/equivocal-avidity levels. All 3 seromarkers were tested using commercial Enzyme Linked Immunosorbent Assays. Data were analyzed by SPSS. Majority (97\%) of the population were positive for CMV-IgG. CMV-avidity was low, equivocal or high in $0.25 \%, 2.75 \%$ and $97 \%$ of IgG seropositives respectively. Age, bad obstetric history and multiparity didn't show association with IgG seropositivity. In conclusion, CMV seroprevalence was high in this study population with low rates of recent primary infection. Education regarding preventive measures for $\mathrm{CMV}$ infection in antenatal care has a value as some proportion were seronegative for $\mathrm{CMV}$ with prone to get new infections.
\end{abstract}

Key Words: CMV, pregnancy, primary infection, IgG- avidity

${ }^{a}$ Consultant Medical Virologist, Virology Department, Medical Research Institute, Colombo 08, Sri Lanka

${ }^{b}$ Consultant Obstetrician \& Gynaecologist, De Zoysa Hospital for Women,

Colombo 8, Sri Lanka

' Senior House Officer, De Zoysa Maternity Hospital, Colombo 8, Sri Lanka

${ }^{d}$ Medical Virology Registrar, Virology Department, Medical Research Institute, Colombo 08, Sri Lanka

${ }^{e}$ Medical Laboratory Technologist, Virology Department, Medical Research Institute, Colombo 08, Sri Lanka

Correspondence: Dr. J. I. Abeynayake MBBS, Diploma Medical Microbiology, MD

Email: janakiiabeynayake@yahoo.com (D) http://orcid.org/0000-0002-3425-7635

Competing interests:The authors report no conflict of interest

\section{INTRODUCTION}

Cytomegalovirus (CMV) is a leading cause for the congenital infections causing severe malformations in the fetus and the newborn with an affected rate of $0.2 \%$ $2.5 \%$ worldwide ${ }^{1}$. It causes intracerebral calcification, ventriculomegaly, microcephaly, sensory neural hearing loss $(\mathrm{SNH})$, retinitis, hepatosplenomegaly and intrauterine growth retardation ${ }^{2}$.

$\mathrm{CMV}$ is a ubiquitous virus with varying seroprevalence worldwide according to the country and population. In developed countries with good socioeconomics, CMV seroprevalence in pregnant population ranges $40 \%-60 \%$ while it is $90 \%-100 \%$ in developing countries ${ }^{3}$. Young children excrete the virus for a longer period and mothers with children or workers of day care centers are at risk for CMV infection ${ }^{4}$.
CMV infection in pregnancy can be categorized in to two types, primary or recurrent infection due to reactivation or re-infection from a different strain ${ }^{5}$. Primary infection during first half of the pregnancy transmits the infection to the fetus in about $30 \%-50 \%$ but only 10 $15 \%$ of infected babies show signs of congenital CMV at birth with $10 \%$ of perinatal mortality. From the $90 \%$ of newborns who are asymptomatic at birth develop symptoms like SNH in later life. In contrast, recurrent $\mathrm{CMV}$ infection carries the rate of newborn infection of about $1 \%$ with the $1 \%-10 \%$ of babies present with symptoms at birth ${ }^{6,7}$.Children with congenital CMV infection following first trimester maternal infection are more likely to have central nervous system sequelae, whereas infection acquired in the third trimester has a high rate of intrauterine transmission but a favorable outcome ${ }^{8}$.

Therefore identification and differentiation of primary CMV infection from recurrent infection in early pregnancy is an important goal in newborn health. As most of maternal infections are asymptomatic, laboratory screening tests for CMV infection play a major role ${ }^{3}$. If mother is diagnosed to have primary infection in early pregnancy serial ultrasound scans (USS) and fetal tests should be done to rule out the congenital infections and malformations. And also there are preventive measures including CMV hyperimmunoglobulin (HIG) and antivirals to reduce congenital malformations which are being evaluating in clinical trials at present. If the mother is found to be seronegative during the screening, mother should be strictly advised regarding hygienic practices like frequent hand washing and avoiding close contact with young children to minimize the acquisition of new infection during pregnancy ${ }^{9,10}$. 
Serological diagnosis of recent viral infection mainly depend on detection of virus specific IgM, but it has several limitations with false positives and unable to differentiate primary from recurrent infection $^{5,}{ }^{11}$. In early phase of CMV infection, avidity of IgG is low and it becomes high during maturation procedure with time. Therefore CMV IgG-avidity can be used to differentiate primary from non primary infection especially in first half of pregnancy ${ }^{12,13}$.

Some European countries offer routine screening to pregnant mothers for CMV infection with well established testing algorithms ${ }^{14}$. In Sri Lanka, still there are no studies and data available on seroprevalence of CMV in pregnancy and burden of congenital CMV infection. The basic data concerning CMV infections during pregnancy is important for health planners and care providers and also the results of this study would add data to the national CMV data bank.

Objective of this study is to screen a selected pregnant population for CMV infection and to describe associated factors with the seropositivity in a major maternity teaching hospital, Sri Lanka.

\section{MATERIALS AND METHODS}

This was a descriptive cross sectional study done for a one year period starting from August 2015. This was included randomly selected 385 pregnant women. Sample size was calculated using WinPepi software to get the $95 \%$ confidence interval by adding $50 \%$ of prevalence data since the local seroprevalence data is not available ${ }^{15}$.

Pregnant patients who were in second trimester coming for antenatal care to the clinics in De Soyza Maternity Hospital, Colombo were included in the study. Pregnant mothers with underlying immunocompromised states such as HIV positive mothers, mothers on immunosuppressive agents were excluded from the study.

Venous blood $(03 \mathrm{~mL})$ was drawn from each eligible mother into a plain container using a $05 \mathrm{~mL}$ disposable syringe under aseptic venipuncture technique. Samples were kept for 1 hour at room temperature to separate the serum and transported to the Virology Reference LaboratoryMedical Research Institute within 6 hours.
All samples were tested for CMV IgG and positive $\mathrm{IgG}$ samples were further analyzed using CMV IgG-avidity assay. CMV IgM test was carried out on the samples negative for CMV IgG and also on samples with low avidity or equivocal results in IgG-avidity according to Illustration 1. All 3 serological markers (IgG, IgG-avidity, and IgM) were tested using commercially validated Enzyme Linked Immunosorbent Assays (ELISA). Euroimmun anti-CMV IgM, IgG and IgG avidity kits were used for testing according to the manufacturer's instructions. Both IgM and IgG kits had a specificity and sensitivity of $99 \%-100 \%{ }^{32}$.

\section{Illustration 1-Testing algorithm for CMV serology}

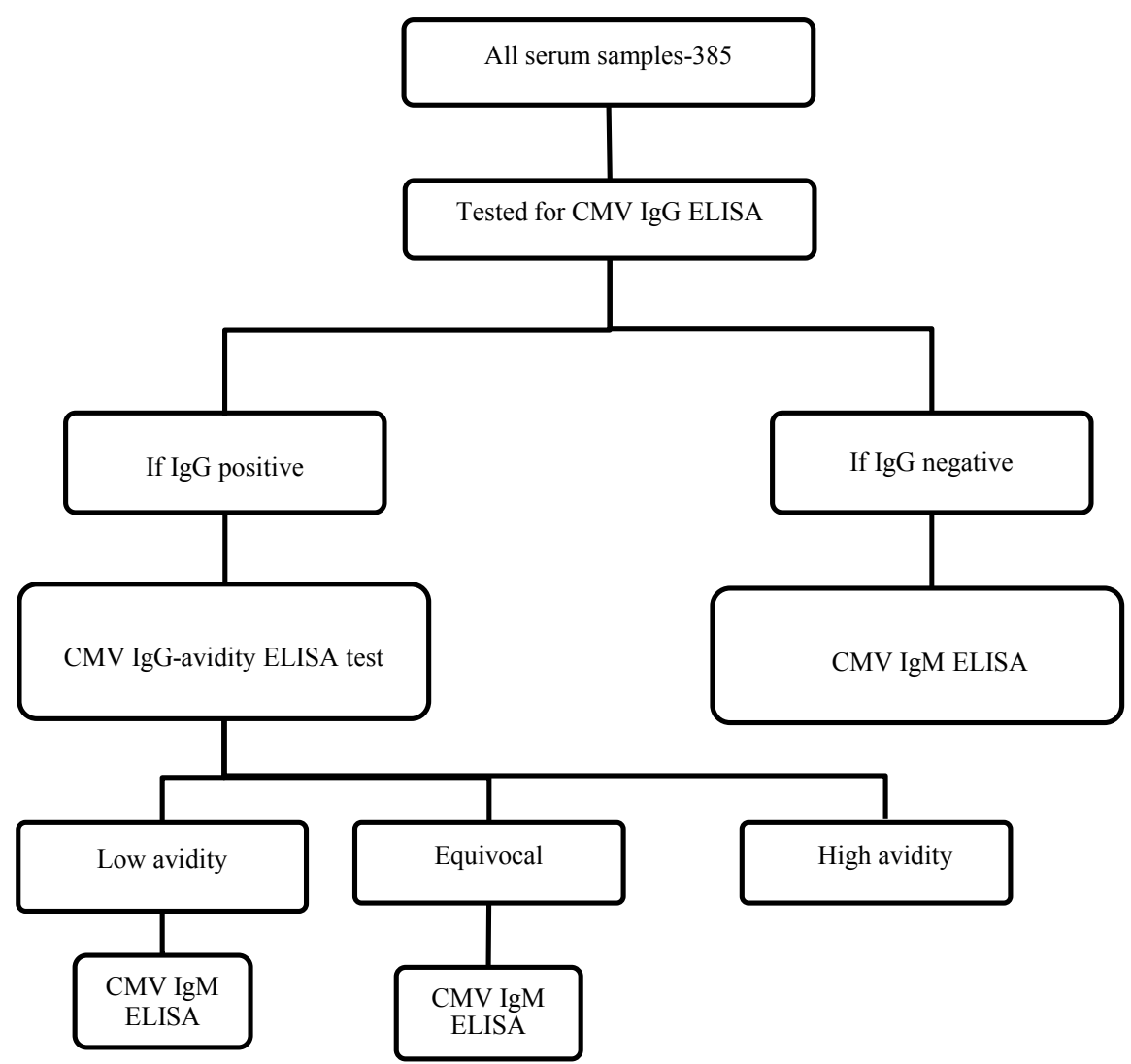

The CMV IgG-avidity test was performed with urea as denaturing agent using commercial diagnostic kit (Euroimmun - ELISA for avidity of CMV-IgG). The IgG Relative avidity index (RAI) was calculated and expressed as percentage by dividing the extinction of the sample with urea treatment by the extinction of the sample without urea treatment. The interpretation of RAI results has been determined as follows: RAI $<40 \%$ indicated low avidity antibodies; RAI 40$60 \%$ indicated equivocal range and RAI $>$ $60 \%$ indicated high avidity antibodies ${ }^{32}$.

Interviewer based questionnaire was used to gather basic sociodemographic data and other information on parity and past medical history by a relevant medical officer before sample collection.

Ethical approval was taken from Ethical review committee, Medical Research Institute. Each study participant was 


\section{RESULTS}

\section{SOCIO-DEMOGRAPHIC AND CLINICAL \\ CHARACTERISTICS}

Total number of 385 pregnant females in their second trimester was enrolled in the study. They were in age range of 16 years -46 years with the mean age of 27 years and standard deviation (SD) of 6.18.

Out of total number of pregnant females, $60 / 365(16.4 \%)$ had a previous bad obstetric history in past with miscarriages, intrauterine death or intrauterine growth retardation

According to the parity, $180 / 385$ (47\%) participants were in their first pregnancy$\mathrm{P}_{1} \mathrm{C}_{0}$ and $21 / 385(5 \%)$ of total were in second or third pregnancy without any previous live births/children- $\mathrm{P}_{2} \mathrm{C}_{0} / \mathrm{P}_{3} \mathrm{C}_{0}$. And rest of the participants- $184 / 385$ $(48 \%)$ was in second or subsequent pregnancies with previous live births/ children.

\section{IgG-AVIDITY ASSAY}

All IgG positive samples were tested for CMV IgG-avidity. From IgG positive samples, only $1 / 374(0.25 \%)$ showed low avidity, 10/374 (2.75\%) showed equivocal results while majority of 363/374 (97\%) showed high avidity IgG.

\section{IgM RESULTS}

CMV IgG negative samples (eleven) and low (one) and equivocal (ten) IgG avidity samples were tested for CMV IgM. All (22/22) were negative for CMV IgM.

\section{DISCUSSION}

This cross sectional study was carried out in a major maternity hospital in Western province, Sri Lanka among pregnant patients of second trimester.

As discussed by Walker et al. 2013, screening for CMV could potentially reduce the burden of congenital CMV in one of three ways. Primary prevention through advising seronegative mothers

Table 1 CMV serology results

\begin{tabular}{|c|c|c|c|c|c|c|}
\hline \multicolumn{2}{|c|}{ CMV IgG } & \multicolumn{3}{c|}{ CMV IgG-avidity } & \multicolumn{2}{c|}{ CMV IgM } \\
\hline positive & negative & high & low & equivocal & positive & negative \\
\hline 374 & 11 & 363 & 1 & 10 & 0 & 22 \\
$(97 \%)$ & $(3 \%)$ & $(97 \%)$ & $(0.25 \%)$ & $(2.75 \%)$ & & $(100 \%)$ \\
\hline
\end{tabular}

\section{IgG RESULTS}

Majority of the study population, 374/385 (97\%) were positive for CMV IgG. Mean age of $\mathrm{IgG}$ positive group and negative group were similar and it was 27 years. There was bad obstetric history in 59/374 $(18 \%)$ of IgG positive group while only $1 / 11(9 \%)$ gave a bad obstetric history in $\mathrm{IgG}$ negative group. 199/374 (53\%) of IgG positive group and 6/11 (54\%) of IgG negative group were in second or subsequent pregnancies with children at home. Chi square test was done to analyze the association of age, bad obstetric history and parity with the CMV IgG positivity. There were no significant association found between these variables and IgG positivity. regarding precautions of hygienic measures is important in minimizing seroconversion. Among women who seroconverted during pregnancy, CMV HIG show significant reduction of perinatal transmission (secondary prevention), and when given to pregnant mother of already infected fetus also have some effect on reducing the risk of malformations (tertiary prevention) ${ }^{9,10}$.

Diagnosis of CMV infection usually relies on serological testing. But confirmation of CMV seroconversion during pregnancy carries several practical challenges. Usually the diagnosis of recent viral infection can be identified by detecting the seroconversion of IgM. However, CMV IgM may found in both primary and recurrent infections and in some patients, IgM may persist for many months after the primary infection ${ }^{16}$. Therefore CMV IgM detection is not specific for diagnosing primary infection and not provides information on whether CMV infection occurred before or after the conception.

Assays based on IgG avidity have the capacity to fill the gaps and provide information on timing of infection. Detection of CMV specific IgG antibody in association with low IgG avidity demonstrates primary CMV infection ${ }^{29}$.

There are various testing algorithms in literature including first to check IgM and if it is positive only, to proceed with CMV IgG-avidity assay. But there are evidence that CMV IgG positive but IgM negative mothers with low avidity $\operatorname{IgG}$ indicating high risk for congenital infection ${ }^{17,18}$. This might be due to the narrow time window with reversion of CMV IgM but still low IgG-avidity. Testing algorithms that direct avidity testing only in IgM positive samples may miss some high risk mothers.For this reason, this study used the algorithm that all sera first tested for CMV IgG and all IgG positive samples to be tested for CMV IgG-avidity with subsequent IgM.

In this study, majority of patients (97\%) were positive for CMV IgG which indicated serological evidence of past CMV infection. Since published data on CMV seroprevalence for pregnant population in Sri Lanka is not available, data of this study is compared with regional and global data.

Seropositivity rates lie between 50\%$60 \%$ in developed countries, and the rates are between $90 \%-100 \%$ in developing countries $^{19,20}$. CMV seropositivity rates are higher in women of reproductive age especially in low socioeconomic status. In different parts of India, serological surveys have shown $80-90 \%$ prevalence of CMV IgG antibodies in women of childbearing age $^{19,20}$. However, in some of European countries, low CMV infection rates have been reported, Australia (56.9\%) and France $(46.8 \%)^{21}$.

Among IgG seropositive group, 18\% had bad obstetric history and $53 \%$ were in second or subsequent pregnancies. Some studies in literature showed significant association of number of parity with IgG seropositivity ${ }^{22}$. Age didn't show significant association with $\operatorname{IgG}$ positivity. In literature there are different concerns on maternal age and CMV infection. Most studies were showed elderly females 
are at a high risk, while others reporting contrary $23,24,25$.

Low affinity CMV IgG are produced in the first 18-20 weeks after infection ${ }^{26,27}$. A subsequent maturation process generates IgG antibodies with higher avidities. Therefore a high IgG-avidity excludes a recent primary infection with low risk of congenital infection. Conversely, IgG -low avidity antibodies together with reactive CMV IgM strongly support the diagnosis of maternal primary infection in the preceding 3 or 4 months ${ }^{26,27}$.

India has higher seroconversion rate (2\%-2.5\%) among pregnant population, whereas only one sample $(0.25 \%)$ showed low affinity antibodies indicating primary infection $^{19,20}$. Equivocal results were found in $2.75 \%$ of IgG positive samples. As equivocal results do not exclude recent infection, all those were tested for CMV $\mathrm{IgM}^{28}$ and all were negative for $\operatorname{IgM}$ suggesting no recent infection with CMV.

Majority of total IgG positive samples (97\%) showed high avidity results suggesting past CMV infection and less risk for the fetus ${ }^{29}$.

IgG negative samples ( $3 \%$ of total study population) were tested for CMV IgM and all were negative. Both CMV IgG and $\operatorname{IgM}$ negativity indicate no serological evidence of CMV infection. And this 3\% of seronegative study population is prone to get a new primary infection in present pregnancy or in subsequent pregnancies. The rate of susceptibility at the beginning of pregnancy varies by the country and also within the country with highest rates occurring among developing countries. Among women of childbearing age, $40 \%-80 \%$ are susceptible (seronegative) to $\mathrm{CMV}$ at the beginning of pregnancy according to available global data ${ }^{30}$.

CMV is transmitted from person to person via close non-sexual contact, sexual activity, breastfeeding, blood transfusions, or organ transplantation ${ }^{31}$. For pregnant women, important sources of infection include sexual activity and contact with the urine or saliva of young children, especially their own children ${ }^{4}$.Therefore these mothers should be counseled regarding the risk and preventive measures including frequent hand washing and avoidance of direct contact particularly with body fluids from preschool children 2. This is more important as $54 \%$ of seronegative mothers of this study were having their own children at home.

\section{CONCLUSION}

This will be the first published study on CMV infection among pregnant women in Sri Lanka and showed maternal CMV IgG seroprevalence at a high level which revealed as $97 \%$. Since there is a naïve cohort for CMV infection among pregnant population, education programmes regarding preventive methods to minimize new CMV infection are important in antenatal care.

However, further studies with large sample volume are needed to analyze with cost effectiveness and feasibility of CMV screening during pregnancy to minimize congenital CMV disease.

\section{REFERENCE}

1. Wong A, Tank KH. Seroprevalence of Cytomegalovirus, toxoplasma and parvovirus in pregnancy. Singapore Med J.2004;41:151-155.

2. Adler SP. Screening for Cytomegalovirus during Pregnancy. Infectious Diseases in Obstetrics and Gynecology.2011;1-9.

3. Uyar Y, Balci A, Akcali A,Cabar C. Prevalence of rubella and cytomegalovirus antibodies among pregnant women in northern Turkey New Microbiologica.2008;31:451-455.

4. Pass RF, Hutto C, Ricks R, Cloud GA. Increased rate of cytomegalovirus infection among parents of children attending daycare centers. N Engl J Med 1986;314:1414-1418.

5. Hodinka RL. Human cytomegalovirus. In: Murray PR, Baron EJ, Jorgensen J, Pfaller MA, Yolken EH, eds. Manual of clinical microbiology. 8th ed., Washington: ASM Press,2003;8:1304-18.

6. Daiminger A, Bader U, Enders G. Pre- and periconceptional primary cytomegalovirus infection: risk of vertical transmission and congenital disease. BJOG 2005;112:166-72.

7. Dangel V, Bader U, Enders G. Improvement of cytomegalovirus avidity test by adjusting the concentration of CMV-specific IgG in test samples. J Clin Virol.2006;35:303-309.
8. Yinon Y, Farine D, Yudin MH. Screening, diagnosis, and management of cytomegalovirus infection in pregnancy. Obstet Gynecol Surv 2010;65:736-743.

9. Walker SP, Palma-Dias R, Wood EM, Shekleton P, Giles ML. Cytomegalovirus in pregnancy: To screen or not to screen. BMC Pregnancy Childbirth 2013;13:96.

10. Vereeck S, Jacquemyn Y. Screening for Cytomegalovirus: An Analysis of Guidelines. J Preg Child Health 2016;3:5.

11. Mace M, Sissoeff L, Rudent A, Grangeot-Keros L. A serological testing algorithm for the diagnosis of primary CMV infection in pregnant women. Prenat Diagn.2004;24:861-863.

12. 12. Revello MG, Gerna G. Diagnosis and management of human cytomegalovirus infection in the mother, fetus and newborn infant. Clin Microbiol Rev.2002;15:680715.

13. Hermann KL, Erdman DD. Diagnosis by serologic assay. In: Lennette EH, Lennette DA, Lennette, eds. Diagnostic Procedures for Viral, Rickettsial and Chlamydial Infections. 7th ed. Washington: American Public Health Association,1996;121-138.

14. Seror J, Bordes P, Luton D. Routine screening for CMV during pregnancy: Practices assessment in Ile-de-France. Gynecol Obstet Fertil 2013;41:578-582.

15. Abramson,J.H.

WINPEPI updated: computer programs for epidemiologists, and their teaching potential. Epidemiologic Perspectives \& Innovations 2011;8:1.

16. Lazzarotto $\mathrm{T}$, Varani $\mathrm{S}$, Gabrielli L, Spezzacatena P, Landini MP. New advances in the diagnosis of congenital cytomegalovirus infection. Intervirology 1999;42:390-397.

17. BaAlawi F, Robertson PW, Lahra M, Rawlinson WD. Comparison of five CMV IgM immunoassays with CMV IgG-avidity for diagnosis of primary CMV infection. Pathology 2012;44:381-383. 
18. Prince HE, Lapé-Nixon M, Brenner A, Pitstick N, Couturier MR. Potential impact of different cytomegalovirus (CMV) IgM assays on an algorithm requiring $\mathrm{IgM}$ reactivity as a criterion for measuring CMV IgG-avidity. Clin. Vaccine Immunol.2014;21:813-816.

19. Chakravarti A, Kashyap B, Matlani M. Cytomegalovirus infection: An Indian perspective. Indian $\mathrm{J}$ Med Microbiol 2009;27:3-11

20. Gandhoke I, Aggarwal R, Lal S, Khare S. Congenital CMV infection in symptomatic infants in Delhi and surrounding areas. Indian $J$ Pediatr 2006;73:1095-1097.

21. Picone O, Vauloup-Fellous $\mathrm{C}$, Cordier AG: A 2-year study on cytomegalovirus infection during pregnancy in a French hospital. BJOG 2009;116:818.

22. Hamdan HZ, Abdelbagi IE, Nasser NM, Adam I. Seroprevalence of cytomegalovirus and rubella among pregnant women in Western Sudan. Virol J 2011;8:217.

23. Bukbuk DN, el Nafaty AU, Obed JY: Prevalence of rubella-specific $\mathrm{IgG}$ antibody in non-immunized pregnant women in Maiduguri, north eastern Nigeria. Cent Eur J Public Health 2002;10:21-23.
24. Rodier MH, Berthonneau J, Bourgoin A, Giraudeau G, Agius G, Burucoa C, Hekpazo A, Jacquemin JL. Seroprevalences of Toxoplasma, malaria, rubella, cytomegalovirus, HIV and treponemal infections among pregnant women in Cotonou, Republic of Benin. Acta Trop 1995;59:271-277.

25. Schoub BD, Johnson S, McAnerney JM, Blackburn NK, Guidozzi F, Ballot $\mathrm{D}$, Rothberg A. Is antenatal screening for rubella and cytomegalovirus justified? S Afr Med J 1993;83:108110.

26. BlackburnNK1,BesselaarTG,Schoub BD, O'Connell KF. Differentiation of primary cytomegalovirus infection from reactivation using the urea denaturation test for measuring antibody avidity. $J$ Med Virol.1991;33:6-9.

27. Manicklal S, EmeryVC, Lazzarotto T, Boppana S B. The "Silent" Global Burden of Congenital Cytomegalovirus. Clinical Microbiology Reviews 2013;26:86102.

28. Prince H E, Lapé-Nixon M. Role of Cytomegalovirus (CMV) IgG Avidity Testing in Diagnosing Primary CMV Infection during Pregnancy. Clinical and Vaccine Immunology 2014;21:1377-1384
29. Duff P. A Thoughtful algorithm for the accurate diagnosis of primary CMV infection in pregnancy. Obstet Gynaec 2007;196:196-197.

30. Colugnati F A, Staras, S A, Dollard $\mathrm{S}$ C, Cannon M J. "Incidence of cytomegalovirus infection among the general population and pregnant women in the United States," BMC Infectious Diseases 2007;7:71.

31. Stagno S. Cytomegalovirus. In Infectious Diseases of the Fetus and Newborn Infant, eds. Remington JS, Klein JO. Philadelphia: W.B. Saunders Company,2001:389-424.

32. Bonalumi S, Trapanese A, Santamaria A, D'Emidio L, Mobili L. Cytomegalovirus infection in pregnancy: review of the literature. Journal of Prenatal Medicine.2011;5(1):1-8. 